\title{
Berlusconi e Macron: figure per esplorare l'ipermoderno Un'analisi de ll Duca di Mantova di Franco Cordelli e Un personnage de roman di Philippe Besson
}

diGerardo landoli

\begin{abstract}
L'articolo analizza II Duca di Mantova di Franco Cordelli e Un personnage de roman di Philippe Besson. In questi testi, gli autori si confrontano rispettivamente con la figura di Silvio Berlusconi ed Emmanuel Macron. Attraverso la categoria di 'ipermoderno', l'articolo intende studiare alcuni tratti del potere contemporaneo: in una prima parte, si analizzerà lo statuto ontologico dei testi e le modalità usate per mescolare elementi di finzione a dati reali; successivamente, si mostrerà quali immagini del potere emergono dai due politici rappresentati; in conclusione, andando oltre i testi, si cercherà di definire il concetto di 'Leviatano-ologramma', al fine di fornire nuovi strumenti concettuali per decostruire l'immaginario politico contemporaneo.
\end{abstract}

ABSTRACT: The article analyses Franco Cordelli's // Duca di Mantova and Philippe Besson's Un personnage de roman. In these texts, the authors compare themselves with the figure of Silvio Berlusconi and Emmanuel Macron respectively. Through the category of 'hypermodern', the article intends to study some traits of contemporary power: first of all, it will analyse the ontological status of the texts and the ways used to mix fictional elements with real data; then, it will show which images of power emerge from the two represented politicians; in conclusion, going beyond the texts, it will try to define the concept of 'Leviathan-hologram', in order to provide new conceptual tools to deconstruct the contemporary political imaginary. 
PAROLE CHIAVE: Franco Cordelli; Philippe Besson; II Duca di Mantova; Un personnage de roman; Ipermodernità; Potere

KEY WORDS: Franco Cordelli; Philippe Besson; Il Duca di Mantova; Un personnage de roman; Hypermodernity; Power

Il presente saggio intende riflettere sulle rappresentazioni del potere contemporaneo, attraverso il concetto di 'ipermoderno'. Tale termine ha avuto una particolare fortuna critica agli inizi degli anni Duemila, grazie principalmente all'opera di due sociologi francesi: Nicole Aubert e Gilles Lipovetsky. La prima ha raccolto le riflessioni di vari autori sul tema, apparsi nel volume L'individu hypermoderne nel 2004. II secondo ha influenzato, con i suoi studi sulla società contemporanea, tali riflessioni: in particolar modo si ricorda un testo pubblicato insieme a Sébastien Charles, anch'esso uscito nel 2004, fondamentale per orientarsi in questa particolare categoria critica: Les Temps hypermodernes. Tutti questi interventi, nonostante coinvolgano varie discipline e approcci (si passa dalla psicoanalisi all'urbanistica), hanno un fattore in comune: si focalizzano sui processi di soggettivazione dell'individuo contemporaneo, condizionati da cambiamenti sociali che iniziano negli anni Ottanta, ma che si affermano nel nuovo millennio (Aubert 15). Nello specifico, il suffisso 'iper' “désigne le trop, l'excès, l'au-delà d'une norme ou d'un cadre. Il entre dans le champ de signification des superlatifs avec une connotation de dépassement constant, de maximum, de situation limite" (Rhéaume 93). La sua fusione col termine 'moderno', quindi, starebbe a indicare una "exacerbation", una "radicalisation de la modernité" (Aubert 14-15). In sintesi, esplorare l'ipermodernità significa studiare le pratiche che gli individui mettono in atto per formare la propria identità, alla luce di questa intensificazione delle caratteristiche che appartenevano all'uomo moderno.

Il focus sui processi di soggettivazione non è casuale, ma ispirato dal pensiero del filosofo politico Marcel Gauchet, altro punto di riferimento per gli studiosi dell'ipermoderno (Gauchet, Conclusion 291-301). Egli ha definito l'individuo contemporaneo "déconnecté", perché rinuncia al punto di vista dell'insieme per osservare il mondo solo dalla prospettiva di ciò che gli interessa (Gauchet, démocratie 254). È interessante notare come, negli stessi anni, anche la letteratura francese si stesse occupando del problema della soggettività: al riguardo, la critica Marie-Odile André, già nel 2001, registrava l'affermarsi di scritture legate al genere biografico e autobiografico, dove i dati reali si mescolavano ad altri fittizi (André 31-47).

Anche in Italia, il concetto di ipermoderno è strettamente legato alle problematiche della soggettivazione. All'inizio della seconda decade del nuovo millennio, infatti, lo psicoanalista Massimo Recalcati usa la categoria di ipermoderno per esplorare le "figure della nuova clinica psicoanalitica" e il critico Raffaele Donnarumma 
per sottolineare come "contro il mito postmoderno della morte del soggetto, la presa di parola individuale è uno dei fenomeni tipici" della letteratura di oggi (Donnarumma, Ipotesi 31). Nel 2014, Donnarumma amplia le sue riflessioni dando alle stampe Ipermodernità: da quel momento, seppur con le dovute critiche (Guglielmi; Policastro), il concetto di ipermoderno, almeno in ambito letterario, trova in Italia una sua collocazione stabile. Daniele Giglioli, citato dallo stesso Donnarumma (Ipermodernità, 238), ha proposto di analizzare i testi contemporanei come "sintomi" del mondo in cui appaiono: "si tratta, le opere, di farle entrare - meglio ancora: rientrare - nel mondo, scorniciandole dal contesto sterilizzante in cui la letterarietà le pone. Non per farne degli enunciati pseudo-referenziali [...], ma per pensarci e sentirci attraverso" (Giglioli 102). Alla luce di queste parole, l'articolo si pone l'obiettivo di riflettere sulle strutture del potere contemporaneo attraverso la letteratura. Nello specifico si studieranno due opere: II Duca di Mantova (2004) di Franco Cordelli e Un personnage de roman (2017) di Philippe Besson. Si è scelto di mettere a confronto queste due opere perché esse presentano alcune affinità strutturali e tematiche. In entrambi i casi, la voce narrante è un io che si identifica con quello dell'autore sulla copertina. Questi io si concentrano sul loro rapporto con una particolare figura politica: Cordelli si misura con Silvio Berlusconi, mentre Besson con Emmanuel Macron. Gli autori abbandonano qualsiasi pretesa di oggettività per dedicarsi a una scrittura profondamente soggettiva, in cui il loro punto di vista è profondamente marcato. Gli scrittori si confrontano con la forza seduttiva dei due presidenti, la quale sembra condizionare il loro modo di scrivere e di pensare. Tuttavia, gli intenti sono molto diversi: I'obiettivo di Cordelli è quello di "disincantare" (Cordelli 115), mentre quello di Besson, amico personale di Macron, è di dare forma a questo 'incantamento': "Je vais écrire une ésperance" (Besson 14).

Fin da adesso è necessario precisare che non si farà riferimento ai due personaggi politici reali, bensì al mito che è scaturito dalle loro figure. Qualsiasi riferimento a fatti reali sarà teso non alla verificazione dei testi, bensì a una maggiore comprensione del mito stesso. Berlusconi e Macron, in queste opere, assumono il ruolo di 'figure', le quali, secondo Roland Barthes, sono "idealità simboliche" (Barthes 66) che incarnano certi valori o processi. D'altronde, Cordelli dichiara: "Non: chi è il Duca di Mantova, ${ }^{1}$ chi se ne frega; ma: che cosa egli significa" (Cordelli 108), mentre Besson, commentando il suo scritto, ha affermato di aver voluto raccontare un momento in cui "I'Histoire s'écrit" (Besson 187), facendo intendere di essere interessato maggiormente alle dinamiche generali, più che agli attori che mettono in moto tali dinamiche. II presente articolo vuole perciò, attraverso la letteratura, individuare i 'valori' rappresentati da queste figure, per poter riflettere, nell'epoca ipermoderna, sul rapporto tra potere e individuo.

In conclusione, si cercherà di valicare i confini della critica letteraria e della critica di questi testi in particolare, per proporre nuovi strumenti di analisi delle strutture del potere contemporaneo.

${ }^{1}$ Lo si approfondirà più avanti: qui basta dire che in Cordelli l'espressione 'Duca di Mantova' designa la figura di Berlusconi.

Fuori verbale/Entre mamparas/Hors de propos/Off the Record

N. $26-11 / 2021$

ISSN 2035-7680 


\section{FAR FINTA DI FAR-FINTA}

Secondo Manfred Jahn, ogni lettore 'affaccia' sul mondo possibile descritto dal testo letterario. Il lettore osserva una particolare porzione del mondo possibile attraverso una specifica lente, incarnata dall'istanza narrativa presente nel testo (Jahn, Windows 241267). Tra i vari tipi di focalizzazione, quella stretta indica una modalità testuale in cui l'angolo visuale del lettore si cala nello sguardo di un particolare personaggio (Jahn, Aspects 85-110). Visto che in entrambi testi presi in esame il narratore si confonde con l'autore, chi legge non viene immerso nel punto di vista di un personaggio fittizio, ma di uno percepito come in carne e ossa. Da questo fattore dovrebbero scaturire degli effetti di reale, che, però, entrambi i narratori cercano di frenare. In Cordelli si legge: "Per quanto riguarda la prima persona, non sto parlando di me-me, ma come è ovvio di un io-me, un io qualsiasi" (Cordelli 29); mentre in Besson: "La question que je me pose n'est pas celle de l'objectivité, je ne prétends pas à aucune objectivité, je me fous de l'objectivité, je ne suis pas journaliste, ni greffier" (Besson 37). Volendo approfondire ancora di più quest'ultimo aspetto, è utile ricercare le definizioni che i narratori stessi danno a ciò che hanno scritto. Ne II Duca si legge: "Perché non scrivere delle proprie idee fisse; non della propria vita ma delle sue ossessioni?" (Cordelli 72). Di fatto, II Duca non ha una trama precisa: racconta la vita di Cordelli intorno ai suoi cinquantanove anni. Il filo conduttore che lega le varie pagine è la figura di Berlusconi, che ritorna ciclicamente nei pensieri del narratore, condizionandone la vita e il pensiero. Secondo Silvia Cucchi, Berlusconi per Cordelli è "una figura di speculazione astratta" che insiste su e persiste nella sua psiche (Cucchi 84). In un altro punto, Cordelli definisce il testo come "la mia summa teologico-politica" (Cordelli 168): alla luce di ciò si può dire che II Duca sia una speculazione filosofica in cui l'immagine di Berlusconi assume i tratti di una metafisica, che non è il reale, bensì ciò che permette di riflettere sul reale stesso.

Invece, Besson scrive: "Je vais au moins écrire une aventure. Une aventure dont j'ignore l'épilogue, mais dont je sais déjà qu'elle sera faite d'étapes, de rebondissements, de péripéties, de risques, d'obstacles, de franchissements d'obstacles, de hasard, de nécessité" (Besson 14). Un personnage è la storia dell'ascesa al potere di Macron durante la campagna elettorale per le presidenziali francesi del 2017, scritta in presa diretta da Besson, che in quanto amico può seguire da vicino i movimenti di Macron e di sua moglie Brigitte. Eppure, il narratore anziché rivolgersi agli strumenti dell'inchiesta o della cronaca, decide di sfruttare gli stratagemmi della messa in intrigo finzionale, come si evince dalla citazione.

Per poter comprendere l'operazione alla base di questi due testi, è opportuno introdurre il concetto di "far-finta". Secondo il filosofo analitico Kendall L. Walton, esso spiegherebbe ogni processo artistico rappresentazionale. L'essere umano, generalmente, sperimenta per la prima volta il far-finta durante l'infanzia: i bambini, ad esempio, in un determinato spazio, possono decidere che gli alberi ricoprano il ruolo dei soldati nemici nel gioco 'fare-alla-guerra'. Attraverso un atto di designazione, l'albero assume un ruolo diverso da quello ricoperto nella realtà. II gioco delimita uno spazio, in cui vigono alcune regole che definiscono cosa si possa fare e il valore degli elementi presenti nello spazio stesso. II gioco si attiva quando si rispettano le regole, 
altrimenti si 'ritorna' nel mondo reale in cui già si è (Walton 249). Gli autori in esame fanno l'esatto contrario: parlano di eventi reali e fanno finta di far-finta. Il reale da loro raccontato viene inserito all'interno dello spazio del 'gioco letterario': il lettore, allora, sarà indotto a cercare le 'regole' di questo far-finta, nonostante i fatti narrati appartengano a uno spazio originariamente non ludico.

I due personaggi in questione nel testo assumono i tratti di personaggi letterari o di funzioni narrative: Berlusconi diventa il Duca di Mantova, espressione che rievoca un personaggio del Rigoletto di Giuseppe Verdi, il quale rappresenta il potere (Cordelli 7); Macron è "celui qui incarne l'ambition dans le récit d'aventures et d'action, celui qui cherche à affronter le monde dans le roman réaliste, celui qui, soumis aux élans et aux affres de la passion, s'invente un destin dans le grand mouvement du romantisme" (Besson 172). Nella complessità del reale, gli autori vanno alla ricerca di 'regolarità': l'ossessione di un uomo per Berlusconi si trasforma in un'astrazione filosofica al fine di "comprendere le ragioni dell'avversario" (Cordelli 167); la cronaca di una campagna elettorale diventa il modello di una storia d'avventura, in cui il protagonista, Macron, è l'eroe. L'evento singolo, una volta inscritto nello spazio del gioco letterario, si trasforma in un esperimento: esso deve restituire uno schema, una formula, una legge. Tuttavia, si è ben lontani dalle scritture saggistiche: queste opere mostrano il gioco nel suo svolgimento, con solo qualche commento a margine. Sarà compito di chi legge individuare le regole del gioco: questi testi non sono manuali, ma resoconti di partite svolte da cui trarre insegnamento.

\section{LE REGOLE DEL GIOCO}

A questo punto, bisogna indagare come questi testi possano essere utili per studiare i processi di soggettivazione ipermoderni. In Cordelli si legge: "Penso forse d'essermi trasformato nel Duca? O aspiro a diventare come lui? $\mathrm{O}$, al contrario, è questo uno scongiuro per non essergli simile?" (Cordelli 167); mentre in Besson: "Je sais parfaitement que le risqué existe que je cède à la séduction, que je sois instrumentalisé, voire manipulé. Peut-être se contente-t-il de me faire travailler à sa propre gloire. Chercherai-je à m'en défendre, en l'égratignant ici ou là? Me laisserai-je faire quelquefois ? souvent ?" (Besson 37). Prendendo in considerazione il pensiero di Michel Foucault, "con il termine potere mi sembra che si debba intendere innanzitutto la molteplicità dei rapporti di forza immanenti al campo in cui si esercitano e costitutivi della loro organizzazione; il gioco che attraverso lotte e scontri incessanti li trasforma, li rafforza, li inverte" (Foucault 82). L'interesse di questi testi sta proprio in questo: non si limitano a rappresentare il presidente, ma mettono in mostra le tensioni tra l'io che scrive e il potere. È in questa tensione che si possono individuare le regole del gioco della politica ipermoderna.

Qui si assiste al fenomeno che Judith Butler ha definito di "soggettivazione/assoggettamento": riprendendo la dialettica servo/padrone di Hegel, la filosofa sostiene che l'individuo sviluppa una propria soggettività confrontandosi con il potere, il quale diventa la "coscienza del servo" (Butler 42). II servo, lottando con il 
padrone, rivendica una propria identità, ma i termini di quest'ultima dipendono dalle pratiche che il padrone usa per assoggettare il servo stesso. "Il potere infatti non solo agisce sul soggetto, ma ne stabilisce l'esistenza" (Butler 51): pertanto, i due scrittori sono consapevoli del costante pericolo di essere assorbiti dall'oggetto del proprio interesse, di trasformarsi in pura voce del potere. I presidenti sono il tema principale di questi testi: la loro (e dei loro narratori) ragione d'essere dipende dal potere stesso. C'è un altro aspetto molto importante di cui tener conto: Berlusconi e Macron sono due presidenti ${ }^{2}$ eletti dal popolo all'interno di un sistema democratico di rappresentanza: "Se gli italiani hanno eletto Duca il Duca è perché il Duca gli è piaciuto" (Cordelli 148); "Emmanuel M. remporte l'élection avec 66\% des voix et plus de 20 millions de suffrages" (Besson 172). Come ha mostrato il filosofo politico Bernard Manin, in un suo importante lavoro sul governo rappresentativo, l'eletto deve possedere un tratto distintivo che viene percepito come superiore alle qualità degli altri candidati. Tale tratto non deve necessariamente rinviare a un sistema di virtù universali, ma solo farlo credere. In sintesi, "I'élection sélectionne des supériorités perçues et des différences réelles" (Manin 190). Nel corso dei paragrafi successivi si cercherà, attraverso i testi, di delineare questi tratti distintivi. Si ricorda, però, che il giudizio che i narratori danno alle qualità dei due presidenti è diverso. Cordelli è chiaro: "Io odio il Duca di Mantova" (Cordelli 102). Al contrario, in una postfazione aggiunta all'edizione del 2018, Besson dichiara: "J'ai aimé écrire sur cette conquête du pouvoir précisément. Parce que j'y ai accompagné un homme qui s'est inventé un destin et qui, dans le même mouvement, a fait l'Histoire" (Besson 187). Se nel primo caso si è di fronte a un rapporto contrastivo tra l'individuo e il potere, nel secondo a uno partecipativo. D'altronde Besson confida: "Je me rends compte que j'ai envie qu'il y arrive. Pour la France ou pour mon livre ?" (Besson 44).

\title{
LA SEDUZIONE DEL BUFFONE
}

Berlusconi viene definito come un produttore di parole e immagini, le quali, per Cordelli stesso, "sono azioni" (Cordelli 106). Ma cosa compiono queste azioni?

\begin{abstract}
Pensavamo [Cordelli e i suoi amici] che tutti dovessero diventare poeti, che la poesia fosse il vessillo della liberazione umana, avevamo questa idea, che gli uomini si debbano liberare. [...] Il Duca di Mantova ha di fatto rovesciato la nostra giovanile utopia poetico-comunista. Sono tutti poeti, o quasi-poeti, o disponibili alla poesia - cioè alla narrazione della propria cosiddetta esperienza, immaginazione compresa: dunque, non vi è più alcun poeta, non ve ne è uno che produce eterne azioni, non vengono prodotte che azioni effimere, peggio: effimere e pretenziose, o effimere e felici di essere spazzate via, di vivere immerse in un persecutorio e numerico presente. (Cordelli 107)
\end{abstract}

2 Si ricorda, però, che, da un punto di vista istituzionale, i poteri dei due presidenti, viste le differenze tra le strutture statali dell'Italia e della Francia, divergono considerevolmente: Berlusconi è stato Presidente del Consiglio dei Ministri, mentre Macron è, nella fase di scrittura di questo articolo, Presidente della Repubblica francese. 
Un commento di Valerio Magrelli sull'epoca berlusconiana può rivelarsi utile per capire il passo citato: "Mezzo paese si è messo nelle mani di qualcuno abilissimo nel sollecitare e solleticare la sua capacità immaginativa" (Magrelli 64). Si può affermare che le azioni berlusconiane siano dei predicati causativi, del tipo 'far-immaginare'. L'atto immaginativo è un particolare atto di produzione, il quale, scrive Jean-Paul Sartre, "mira nella sua corporeità a un oggetto assente $o$ inesistente attraverso un contenuto [...] psichico" e dove si trasferiscono "al contenuto psichico trascendente l'esteriorità, la spazialità e tutte le qualità sensibili della cosa" (Sartre 84,85 ). In sostanza, si può dire che l'azione berlusconiana faccia produrre assenze.

Berlusconi è in sintesi, secondo Cordelli, un "cattivo poeta" (107). E tale forma d'arte 'cattiva' sembra essere connaturata al personaggio fin dalle origini: al riguardo, Cordelli descrive l'incontro di sua madre con il giovane Berlusconi, quando ancora sconosciuto lavorava come cantante sulle navi da crociera. Berlusconi, afferma la madre di Cordelli, era "un tipo speciale [...], un intrattenitore" (87). Si può dire che il 'cattivo poeta' sia l'uomo delle televisioni commerciali, dove la leggerezza dell'intrattenimento viene sfruttata per vendere i prodotti delle pubblicità. Come ha notato lo storico Paul Ginsborg, il successo di Berlusconi dipende dal modello di vita proposto dalle sue televisioni, legato al "consumismo, con i suoi cicli perpetui di desiderio - acquisto - uso - delusione - rifiuto - risorgere del desiderio" (Ginsborg 29). L'immagine del 'cattivo poeta' sembra essere efficace per descrivere il mondo economico contemporaneo: la poiesis delle assenze è coerente con la prospettiva lacaniana, secondo la quale il capitalismo propone "il consumo come una strada per colmare il vuoto su cui il desiderio in quanto tale si attiva" (Magatti 132). Berlusconi non vende oggetti di consumo, ma il consumismo stesso. Vende quel vuoto su cui si attivano le speranze e i desideri del pubblico. È in questo aspetto che si situa la forza di Berlusconi: Cordelli, rispondendo alla madre che parla del Berlusconi intrattenitore, aggiunge:

Non si è limitato a fare l'intrattenitore. Subito mi correggo, come prima ho corretto lei: era un animatore, come adesso - sul palcoscenico del mondo. Anzi, un animatore è riduttivo. Egli è un autore postmoderno, ha riciclato gli italiani, ha rifatto I'Italia, I'ha rapita nel senso antico e nobile, cioè metaforico, e poi è stato costretto a per così dire stuprarla, ma le ha restituito la verginità. Benché non si sia decisa a donare i vecchi abiti ai ciechi, I'Italia di oggi è nuova di zecca. (Cordelli 87)

Su questo punto, il mass-mediologo Pierre Musso ha notato come la televisione di Berlusconi sia stata capace di trasferire nella sfera delle attività individuali processi del mondo del mercato. Così, l'intimità stessa è entrata a far parte delle dinamiche commerciali (Musso, Télé-politique 69). Berlusconi incarna l'immagine di un mondo economico che affabula l'individuo attraverso l'intrattenimento, tanto da modificare nel profondo le sue condotte di vita.

Per ampliare il discorso sulla forza attrattiva di Berlusconi, è rivelatrice una delle scene finali del testo, dove Cordelli descrive l'unico momento in cui ha avuto l'occasione di vedere di persona il politico. Durante una partita del Milan, Cordelli riesce a vederlo da lontano sugli spalti. Berlusconi si mostra sorridente al pubblico, "come un attore del cinema" (Cordelli 208). Semplicemente osservandolo, l'autore capta la "femminile 
sostanza" di Berlusconi: "è un travestito; ciò che sommamente di lui ci disturba è la sua ostinazione a non gettare la maschera, che pure gli assomiglia" (Cordelli 209). Al fine di comprendere meglio la natura della femminilità di Berlusconi, è utile introdurre uno studio di Baudrillard sulla seduzione, considerata dal filosofo come una forza femminile che si contrappone a quella maschile della produzione (Baudrillard 29). La seduzione non riguarda la sessualità: infatti, quest'ultima è una forza procreatrice o produttrice di piacere (Baudrillard 71-72), mentre la seduzione è una forza della reversibilità: essa rapisce ed esaurisce la forza altrui (Baudrillard 140). Berlusconi, avendo una sostanza femminile, può essere considerato una figura della seduzione. Alla luce di ciò, si può interpretare la riflessione di Cordelli sul Berlusconi cattivo poeta in questo senso: egli rapisce la forza produttrice dei poeti delle "eterne azioni", dando vita a dei poeti senza opera.

Questo ragionamento può essere ulteriormente approfondito: la scena della partita, così come il capitolo, si conclude con un'invocazione a un dio: "non ci sono alternative, o dio è un sadico o, se c'è, è un essere inutile, che il male non lo può impedire né limitare, né abolire" (Cordelli 209). Tutto ciò può essere messo a confronto con un passo precedente: "Che tipo di autore è il Duca? Qual è l'anima che ci propone, ci impone, ci soffia dentro? Il suo è il mondo magico. Ma noi sappiamo benissimo che gli sciamani sono dei posseduti. Chi dunque lo possiede? Lo possiede un dio o chi altro?" (Cordelli 87). Berlusconi è lo sciamano di un dio impotente: il suo far-immaginare induce visioni mistiche che svuotano l'individuo, gettandolo nell'inazione dell'effimero.

In Berlusconi, afferma Cordelli, "il Re e il Buffone sono la stessissima persona" (167). La figura del Re e del Buffone sono riprese dall'opera di Victor Hugo Le roi s'amuse, fonte del Rigoletto di Verdi. II re François è "la gloria, la sovranità, una specie di perfezione terrestre", mentre il buffone Triboulet è "I'umana facoltà critica, il comico, la forza che viene dai bassifondi" (18). Commentando l'opera di Hugo, Cordelli mostra come "il motore che mira a distruggere la misura, il centro, la Legge" sia ciò che crea l'intrigo dell'intera vicenda (19). Per comprendere il pensiero di Cordelli, potrebbe essere utile fare riferimento agli studi dell'antropologo politico Georges Balandier: mettendo a confronto non solo le fonti storiche, ma anche le rappresentazioni teatrali della figura del buffone (tra cui quella di Triboulet di Hugo), Balandier descrive quest'ultimo come colui capace di rappresentare, con la sua arte, la regressione selvaggia che si realizzerebbe in una società senza norme (64). L'unione di Re e Buffone potrebbe significare allora il desiderio di porre al potere la sfrenatezza stessa. II 'cattivo poeta' è un autore che crea storie sfruttando la forza narrativa della distruzione della Legge: ma mentre il Buffone crea storie per mettere in crisi il potere, il Re/Buffone le crea per confermare tale potere e accrescerlo. Inoltre, Cordelli scrive: "i miei sentimenti sono freddi, l'epoca è fredda, la realtà che qui si manifesta non ha nulla che susciti l'eventuale bestia - che poi sarebbe proprio il Duca" (96). Berlusconi è la figura che rapisce la forza produttiva dell'individuo, al fine di presentarsi come l'unico uomo capace di ripristinare una forza selvaggia, al di là di ogni norma. La televisione getta l'individuo nell'inazione: essa crea il bisogno del movimento, bisogno che viene subito soddisfatto attraverso le immagini, fonte illusoria di possibili attività future. E uno di questi 'sogni di attività' è incarnato dalla figura di Berlusconi stesso. 


\section{LA SEDUZIONE DELLA MACCHINA}

È interessante mettere a confronto questi due testi anche perché permettono di osservare alcune variazioni all'interno della stessa ipermodernità. Se Berlusconi simboleggia il primo decennio degli anni Duemila, in questo paragrafo si cercherà di mostrare come Macron rappresenti il secondo attraverso caratteristiche che continuano e allo stesso tempo superano il decennio precedente.

Berlusconi rappresenta ancora il paradosso ipermoderno evidenziato da Gilles Lipotevsky nel 2004: l'epoca ipermoderna eredita l'edonismo postmoderno, ma lo fa convivere con l'ansia e un senso di vulnerabilità (Lipovetsky 63). Con Berlusconi si spiega questo paradosso: l'edonismo resta sotto forma di illusione, mentre l'individuo è costretto a vivere un costante svuotamento. Andando oltre il testo di Cordelli (si ricorda che è stato pubblicato nel 2004), sono interessanti le riflessioni della giornalista Ida Dominijanni sulla caduta politica di Berlusconi avvenuta nel 2011: alla fine del suo mandato, è ormai un uomo che cerca in tutti i modi di celare, alterandolo, il proprio corpo, che invecchia e diventa debole. Berlusconi incarna il paradosso ipermoderno: l'immagine edonistica del potente attorniato dal suo harem, il bunga-bunga, nasconde in realtà "una potenza sessuale truccata" (Dominijanni 203). Con l'avvento della crisi dei subprime del 2007, l'illusione edonistica non regge più: Berlusconi decade, lasciando spazio a Mario Monti, colui che ha rappresentato, secondo Mario Perniola, nel bene o nel male, "i miti dell'efficienza tecnocratica" (11). Berlusconi lascia una "società disperata" (Perniola 30), alla ricerca di nuove virtù che possano realizzare un miglioramento futuro.

Besson mostra come il mito macroniano nasca proprio in un contesto di crisi come quello appena descritto e si sviluppi nel solco lasciato dalla disillusione nei confronti delle promesse dei decenni precedenti. Le parole di Macron riportate da Besson sono chiare su questo punto: "Notre civilisation peut disparaître, elle est peut-être déjà morte. Je suis peut-être le dernier des Aztèques qui gigote. Les petites compromissions et les pratiques disciplinaires, ça n'était plus à la hauteur de la situation. J'ai préféré partir et prendre le risque de périr" (Besson 13). Addirittura, per Besson è Macron stesso a essere figlio delle contraddizioni del periodo di crisi economica attuale:

Je songe : Emmanuel M. est un enfant de la crise. II n'a jamais connu la croissance, jamais la prospérité. II est né après les Trente Glorieuses. II a grandi dans le chômage de masse, la précarisation galopante. II a vu la France perdre peu à peu son rang de puissance de premier plan et s'accrocher à ce souvenir flatteur. II ne sait rien d'un pays qui irait bien, d'une nation optimiste. Cela explique, en grande partie, son état d'esprit. Mais dans le même temps, Emmanuel M. n'est pas un enfant de la guerre. II a toujours connu la paix. (Besson 52)

La figura di Macron si può capire solo se si tiene conto del fatto che il secondo decennio degli anni Duemila è troppo 'disilluso' affinché la promessa edonistica berlusconiana possa ancora avere potere seduttivo. Macron, per forza di cose, deve

\footnotetext{
${ }^{3}$ In francese, "les Trente Glorieuses" indica il periodo che va dal 1945 al 1975, anni di forte crescita economica per la Francia.
}

Fuori verbale/Entre mamparas/Hors de propos/Off the Record

N. $26-11 / 2021$

ISSN 2035-7680 
rappresentare qualcosa di diverso. Per comprendere il nuovo stile macroniano, una scena è particolarmente significativa: si tratta dell'incontro fortuito avvenuto tra Marine Le Pen e Macron alla fabbrica della Whirlpool ad Amiens, dove gli operai erano in sciopero contro la delocalizzazione:

On se dit alors qu'on a assisté à deux manières radicalement différentes de concevoir la politique: d'un côté, celle de Mme Le Pen, consistant à faire des photos, sur un parking, avec des militants et des nervis, tenant des promesses mensongères, restant dix minutes et se félicitant de son coup de com', de l'autre celle d'Emmanuel M., dialoguant plus d'une heure avec les syndicats, allant au fond des dossiers, allant ensuite au contact des ouvriers, écoutant leur colère, y répondant point par point, pied à pied, disant la vérité, même cruelle. (Besson 164)

Il parallelismo è abbastanza semplice: da una parte c'è una politica dell'immagine (le foto), che illude (false promesse) e dall'altra una politica dei discorsi, del dialogo, addirittura della verità. II mito macroniano si mostra come un'alternativa alla politica della vacuità. Macron "entend incarner le contraire de l'impuissance, le contraire du fatalisme" (Besson 39). Il contrario dell'impotenza, in questo caso, non combacia con la sfrenatezza berlusconiana: ad esempio Macron, nella scena in cui riflette con Besson sulla sua possibile candidatura, afferma:

II faut aussi que je sois totalement prêt dans ma tête. Ça doit s'imposer comme une évidence. [...] Le plus difficile, c'est de trouver l'équilibre entre la lucidité et une volonté qui ne laisse aucune part au doute... Si je manifeste le moindre doute, je suis mort... [...] Chaque jour, des gens disent du mal de moi. Je ne dois rien répondre, rien montrer en retour. Je dois leur offrir le visage impavide de saint Sébastien chez les primitifs italiens, le visage de la Pietà. Rien ne doit transparaître... [Mon entreprise] est Hernani. Une force qui va. (Besson 35)

Questo atteggiamento è coerente con quanto rilevato da alcuni commentatori politici: Régis Debray parla di "néo-protestantisme" del nuovo potere incarnato da Macron, dove al motto "Liberté, Égalité, Fraternité" bisogna affiancare quello di "probité, chasteté, sobriété" (Debray 31). Macron controlla in maniera metodica la sua vocazione politica, il suo Beruf, così come il capitalista descritto da Max Weber controllava il suo stato di grazia attraverso una vita ascetica, dove il controllo razionale di ogni singolo gesto comportava un'assoluta dedizione alla propria professione (Weber 213). II già citato Musso fa notare come il nome del partito politico fondato da Macron, En Marche, assomigli all'espressione francese ça marche, che indica il corretto funzionamento di un dispositivo (Musso, Le temps 75). L'ipermodernità di Macron, allora, risiede in un'estremizzazione dello spirito capitalistico 'protestante'. Alcun dubbio, volontà ferrea, volto impassibile: sono espressioni di un'efficienza coltivata al limite del parossismo, come se si dovesse aspirare a essere una macchina. Significativo quando Besson evidenzia il paradosso di Macron: è un uomo che seduce tutti, ma che resta senza veri amici (Besson 105). La seduzione berlusconiana era calda: il suo sorriso, secondo Marco Belpoliti, era mosso dall'imperativo del "fare sempre simpatia" (Belpoliti 60). La seduzione macroniana, invece, è fredda. A questo punto, è interessante proporre il punto di vista di uno dei più accesi critici di Macron, il saggista e attivista Juan Branco: 
quest'ultimo descrive Macron come un uomo "programmé pour réussir et non construire, propulsé trop vite par un système méritocratique sclérosé par sa richesse et obsédé par la reproduction du même" (Branco 12). Besson e Branco mostrano due atteggiamenti completamente opposti: da una parte un amico di Macron, il quale scrive un libro che ha tutta l'intenzione di celebrare la vittoria elettorale del giovane presidente; dall'altra colui che ha scritto uno dei pamphlet più agguerriti contro Macron. Eppure, è sintomatico notare come, in entrambi i casi, il dato registrato è quello della freddezza e del calcolo: la differenza è che se in Besson tale freddezza è sinonimo di determinazione e quasi di solitudine tragica, in Branco è prova di un assoggettamento alle dinamiche del capitalismo contemporaneo.

Quest'ultimo aspetto messo in evidenza da Branco può essere approfondito prendendo in considerazione un dettaglio, che però diventa rilevante perché è un ulteriore elemento in comune col testo di Cordelli. Si è visto come ne II Duca Berlusconi sia descritto come un 'cattivo poeta' e un 'autore postmoderno'. In Besson, invece, l'aspirazione di Macron alla scrittura è descritta come qualcosa di reale:

(Emmanuel M. a confié, que, dans sa prime jeunesse, il avait l'ambition de devenir écrivain. Son rêve, c'était l'écriture. Pas la finance. Pas la politique. Pas le pouvoir. L'écriture. II a, du reste, " commis » quelques romans dont les manuscrits dorment dans un tiroir, et que sa femme a lus et jugés, semble-t-il, prometteurs mais inaboutis. S'il avait persévéré, il ne serait sans doute pas engagé aujourd'hui dans cette course folle. S'il n'a pas persévéré, c'est sans doute qu'il n'était pas écrivain). (Besson 54)

Questo dettaglio viene scritto tra parentesi: appare come un semplice aneddoto curioso, un modo per spezzare il ritmo del racconto, mentre Besson narra dei preparativi per la prima pubblicazione di Macron, il testo Révolution, di carattere propagandistico. Testo che conferma almeno la grande passione per la letteratura del giovane Macron, nel capitolo in cui il futuro presidente scrive una sintetica autobiografia (Macron 20). Questo semplice racconto tra parentesi acquista maggiore valore se si tiene conto del finale di Un personnage:

«Au fait, Emmanuel, pas de regret de ne pas être devenu écrivain ? » Sa réponse fuse : «La vie n'est pas finie ».

Et il sourit. (Besson 186)

Il testo si conclude così, trasformando quello che era un semplice aneddoto in una promessa, in un destino ancora incompiuto ma di là da venire: se Macron ha avuto successo con la sua campagna elettorale (il testo termina proprio con la vittoria della stessa), allora ci sono le premesse affinché egli possa brillare anche in altri campi. Tuttavia, questo finale a effetto nasconde in sé quello che è il problema del Macron bessoniano: egli è un personaggio potenziale. Questo romanzo trasforma in un'avventura la campagna elettorale, ma non dice nulla dell'attività di governo di Macron: di fatto, il testo viene pubblicato poco dopo le elezioni. Si descrive Macron come un uomo capace di conquistare il potere, ma non si sa nulla di come lo eserciterà. E non sempre le qualità espresse durante la conquista del potere si concretizzano in una 
efficace azione di governo: tutto ciò viene lasciato all'inferenza del lettore, col rischio però che la realtà possa screditare molto presto l'immagine costruita da Besson. Nel finale, l'ormai presidente già pensa a cosa potrà essere in futuro: se Un personnage è la storia di un potenziale presidente che riesce a realizzare il suo intento, il finale mostra un presidente come un potenziale scrittore. L'immagine del mito macroniano di Besson sembra ricalcare ciò che il filosofo Pierre Dardot e il sociologo Christian Laval hanno descritto come "ultrasubjectivation". L'ultrasoggettivazione è un processo di costruzione del proprio sé riscontrabile nell'individuo neoliberale: quest'ultimo cerca costantemente di oltrepassarsi, di non accontentarsi del proprio stato per cercare sempre nuove mete da raggiungere, nuove conoscenze da acquisire, nuove abilità da sviluppare. L'identità del singolo, allora, non risiede più in ciò che si è, ma in ciò che si prefigura di se stessi (Dardot et Laval 437).

II Berlusconi autore proietta sogni edonistici grazie alle proprie televisioni: l'individuo osserva e spera di diventare come quelle immagini. II Macron scrittore potenziale produce da sé il proprio sogno: un individuo instabile che è continuamente proiettato verso un'immagine di sé ancora da realizzare. II dispositivo di potere di Berlusconi, la televisione, è esterno, anche se influenza in tutti i modi l'intimità del singolo. In Macron si assiste a un ulteriore passaggio, che si può comprendere attraverso le parole del filosofo Byung-chul Han: "L'io come progetto, che crede di essersi liberato da obblighi esterni e costrizioni imposte da altri, si sottomette ora a obblighi interiori e a costrizioni autoimposte, forzandosi alla prestazione e all'ottimizzazione" (Han 10). Il continuo desiderio di altro è un dispositivo introiettato nell'individuo contemporaneo, che si assoggetta a una continua ansia da prestazione, perché ormai incapace di godere dello status acquisito. Così come il Macron bessoniano, che appena vinte le elezioni già si proietta verso un avvenire da scrittore.

\section{IL LEVIATANO-OLOGRAMMA}

Besson, seppur applicando alcuni tagli, riporta le parole di un'intervista di Macron rilasciata al giornale Challenges (Domenach, Nicholas, et al.):

François Hollande ne croit pas au président jupitérien. [...] Pour moi, la fonction présidentielle dans la France démocratique contemporaine doit être exercée par quelqu'un qui, sans estimer être la source de toute chose, doit conduire la société à force de convictions, d'actions et donner un sens clair à sa démarche. Quand le président devient «normal», nous courons un risque politique et institutionnel, mais aussi un risque psychologique collectif, et même un risque pour l'efficacité de l'action. (Besson 42)

Subito dopo, Besson commenterà: "L'homme a une parfaite compréhension de l'inconscient monarquique français" (43). L'espressione président jupitérien rinvia a quella di "dio mortale" che Thomas Hobbes usa per descrivere il suo Leviatano, immagine utile per comprendere anche I'inconscio monarchico' evocato da Besson. II Leviatano è un corpo possente, formato dai piccoli corpi dei sudditi. Su di essi svetta il capo, che rappresenta il sovrano. II Leviatano è quindi l'immagine dello Stato, forte 
perché tutti i sudditi decidono di donare il potere a una sola figura, la quale avrà il compito di conservare la pace e di difendere la comunità dai nemici esterni (Hobbes 182). Il presidente Giove, evocato da Macron, sembra rispecchiare la solennità di questa figura. Per approfondire questo aspetto, affiancherei alla citazione di Besson una da II Duca, dove il Leviatano è esplicitamente citato: "La televisione, il Leviatano, proprio perché non produce che gnomi, sia pure gnomi-demiurghi, gnomi-apprendisti stregoni, gnomi-mito, è il campo di battaglia. II Leviatano lo si può affrontare" (Cordelli 137). Qui il Leviatano non è il presidente, ma la televisione, cioè quel dispositivo che ha reso Berlusconi l'uomo potente che è stato. Il governo, quindi, non sembra essere più esercitato dagli uomini, bensì dalla tecnica. Di fatto, si è visto come Macron si descriva efficiente quanto una macchina. II proliferare dei dispositivi nella quotidianità contemporanea, come ha mostrato Giorgio Agamben, attua dei "processi di desoggettivazione" che generano "corpi inerti": "di qui l'eclisse della politica, che presupponeva dei soggetti e delle identità reali [...] e il trionfo dell'oikonomia, cioè di una pura attività di governo che non mira ad altro che alla propria riproduzione" (Agamben 32).

Quanto appena visto è tanto più interessante si si tiene conto del fatto che Bruno Latour ha individuato nel Leviatano di Hobbes uno dei testi costitutivi della modernità, insieme agli studi di Robert Boyle. II primo ha istituito una teoria politica non facendo più riferimento a forze trascendenti, naturali o divine, ma affidandosi soltanto alla forza del ragionamento logico. Il secondo, al contrario, non rinuncia alla trascendenza della natura, ma afferma che è possibile studiarla solo tramite ragionamenti frutto di esperienze umane (gli esperimenti) (Latour 23-70). Da una parte si ha una politica profondamente radicata nel reale, ma descritta con metodi astratti; dall'altra una natura divinizzata, ma studiata attraverso esperimenti concreti. L'ipermodernità di Berlusconi e Macron mescola le carte: la politica fa riferimento a una forza trascendente (il mercato), ma si esprime attraverso l'esperienza del singolo, coerentemente con quanto studiato dalla filosofa Giuliana Parotto. Infatti, oggi si riduce la complessità del discorso politico a "semplici episodi di drammi (o tragedie) messe in scena" (Parotto 11) dai leader. I due testi presi in esame sono figli di questa situazione.

In conclusione, chi scrive intende allontanarsi dall'analisi dei due testi per proporre una nuova figura, nella speranza di illuminare le modalità del potere ipermoderno. Si vuole parlare, quindi, del Leviatano-ologramma. L'ologramma è un'immagine composta da tante unità più piccole: ognuno di questi punti contiene in sé le informazioni sull'intera figura. Se si prova a scindere l'ologramma, anziché avere un'unica figura spezzata, si otterranno tante versioni più piccole dell'intero. La figura non si scompone, anzi: si moltiplica rimpicciolendosi. Se il vecchio Leviatano era composto dai tanti piccoli corpi dei sudditi, il nuovo Leviatano-ologramma è un fascio di luce proiettato dal sistema tecnico-economico. II dualismo sovrano/suddito viene sostituito da un rapporto triadico: i corpi del sovrano e dei sudditi non sono più indipendenti, poiché la loro presenza si fonda sulle proiezioni fantasmatiche introdotte dal dispositivo economico. Allora, il sovrano e i sudditi non saranno nient'altro che punti dell'ologramma, cioè punti che racchiudono in loro tutte le informazioni del sistema. II sovrano perde il suo ruolo di guida, per diventare mero esecutore del dispositivo 
economico. La forza del Leviatano ipermoderno non è generata dai corpi, ma dal vuoto: il capo non simboleggia più un potere che si esercita qui e ora, ma un desiderio da realizzare nel futuro, coerente con quanto previsto dalle regole del mercato. II potere non si radica più nel territorio da governare, ma nell'utopia - edonistica o tecnologica che sia - che esiste solo in quanto luogo proiettato, tele-visivo, ancora non presente. L'ologramma è ben visibile, ma inconsistente al tatto.

II Leviatano-ologramma vorrebbe essere una figura utile per decostruire l'immagine dell'uomo forte al comando: come ha rilevato la sociologa Maria Giuseppina Pacilli, negli ultimi anni si è diffusa l'idea che le questioni politiche debbano essere affrontate da capi che facciano a meno del dialogo con gli altri organi istituzionali e che impongano il proprio volere con rapidità e fermezza (Pacilli 140). A tutto ciò si può aggiungere quanto scritto da Emilio Gentile: analizzando la storia del rapporto tra capi e folla, lo storico ha proposto di definire la democrazia contemporanea come "recitativa" (Gentile 205), intendendo con questa espressione un sistema in cui la massa diventa una mera spettatrice, limitandosi ad applaudire o a fischiare le decisioni dei potenti. Si delinea così l'immagine di un politico che persegue unicamente la propria volontà, scontrandosi in maniera netta con le altre istanze politiche e agendo indipendentemente dalla società civile: in questo tipo di narrazione, il leader è un eroe che persegue unicamente l'affermazione di sé. Eppure, nel corso dell'articolo si è cercato di mostrare come la figura dell'uomo forte al potere, a volte, sia in realtà soltanto un'illusione, poiché il capo del Leviatano-ologramma non è che l'individuazione di un modo di essere imposto dal mondo tecno-economico. II presente studio vorrebbe essere un invito a individuare negli uomini e nelle donne di potere, considerati eccezionali, tutti quegli atteggiamenti che, in realtà, nascondono modi di essere che più che appartenere alla volontà del singolo, sono espressione di meccanismi sociali influenzati dal sistema neoliberale. Un modo per depotenziare la loro aura eroica, per sviluppare uno spirito critico capace di difendere dal fascino che queste figure possono suscitare.

\section{BIBLIOGRAFIA}

Agamben, Giorgio. Che cos'è un dispositivo? Nottetempo, 2006.

André, Marie-Odile. "La littérature française contemporaine: un panorama." Littérature contemporaine en bibliothèque, sous la direction de Martine Poulain, Éditions du Cercle de la Librairie, 2001, pp. 31-47.

Aubert, Nicole. "Un individu paradoxal." L'individu hypermoderne, sous la direction de Nicole Aubert. 2004. Érès, 2010, pp. 11-24.

Balandier, Georges. Le pouvoir sur scènes. Fayard, 2006.

Barthes, Roland. S/Z. 1970. Traduzione di Lidia Lonzi, Einaudi, 1973.

Baudrillard, Jean. De la séduction. 1979. Denoël, 1989.

Belpoliti, Marco. Il corpo del capo. Guanda, 2009.

Besson, Philippe. Un personnage de roman. 2017. Édition enrichie d'une postface inédite de l'auteur, Julliard, 2018. 
Branco, Juan. Contre Macron. 2017. Édition revue et corrigée, Plon, 2019.

Butler, Judith. La vita psichica del potere. 1997. Traduzione di Federico Zappino, Mimesis, 2013.

Cordelli, Franco. /l Duca di Mantova. Rizzoli, 2004.

Cucchi, Silvia. "Lo spettro di Berlusconi: Vasta, Cordelli, Siti." Spectralités dans le roman contemporain. Italie, Espagne, Portugal, sous la direction de Marine Auby-Morici et Silvia Cucchi, Presses Sorbonne Nouvelle, 2017, pp. 79-89.

Dardot, Pierre, et Christian Laval. La nouvelle raison du monde. 2009. La Découverte, 2010.

Debray, Régis. Le nouveau pouvoir. Cerf, 2017.

Domenach, Nicolas, et al. "Macron ne croit pas «au président normal, cela déstabilise les Français »." Challenges, 16 oct. 2016. https://www.challenges.fr/electionpresidentielle-2017/interview-exclusive-d-emmanuel-macron-je-ne-crois-pas-aupresident-normal_432886. Consultato il 15 giu. 2021. 2014.

Dominijanni, Ida. II trucco. Sessualità e biopolitica nella fine di Berlusconi. Ediesse,

Donnarumma, Raffaele. "Ipermodernità: ipotesi per un congedo dal postmoderno." Allegoria, n. 64, 2011, pp. 15-50.

---. Ipermodernità. II Mulino, 2014.

Foucault, Michel. La volontà di sapere. 1976. Traduzione di Pasquale Pasquino e Giovanna Procacci, Feltrinelli, 1988.

Gauchet, Marchel. La démocratie contre elle-même. 2002. Gallimard, 2009. ---. "Conclusion : vers une mutation anthropologique?" L'individu hypermoderne, sous la direction de Nicole Aubert, Érès, 2010, pp. 291-301.

Gentile, Emilio. Il capo e la folla. 2016. Laterza, 2017.

Giglioli, Daniele. Senza trauma. Quodlibet, 2011.

Ginsborg, Paul. Berlusconi. Einaudi, 2003.

Guglielmi, Angelo. "Ipermodernità." Alfabeta2, 14 lugl. 2014. https://www.alfabeta2.it/2014/07/14/ipermodernita/. Consultato il 15 giu. 2021.

Han, Byung-Chul. Psicopolitica. 2014. Traduzione di Federica Buongiorno, Nottetempo, 2016.

Hobbes, Thomas. Leviatano. 1651. Traduzione di Gianni Micheli. Rizzoli, 2016.

Jahn, Manfred. "Windows of Focalization: Deconstructing and Reconstructing a Narratological Concept." Style, n. 30. Vol. 2, 1996, pp. 241-267.

---. "More Aspects of Focalisation: Refinements and Applications." Recent Trend in Narratological Research, edited by John Pier, PUFR, 1999, p. 85-110.

Latour, Bruno. Nous n'avons jamais été modernes. 1991. La Découverte, 2015.

Gilles Lipovetsky, "Temps contre temps ou la société hypermoderne." Les temps hypermodernes par Gilles Lipovetsky et Sébastien Charles. 2004. Grasset, 2013.

Macron, Emmanuel. Révolution. 2016. Pocket, 2017.

Magatti, Mauro. Libertà immaginaria. Feltrinelli, 2009.

Magrelli, Valerio. Il Sessantotto realizzato da Mediaset. Einaudi, 2011.

Manin, Bernard. Principes du gouvernement représentatif. 1995. Flammarion, 2019. Musso, Pierre. Télé-politique. L'Aube, 2009. 
---. Le temps de l'État-Entreprise. Fayard, 2019.

Pacilli, Maria Giuseppina. Uomini duri. II Mulino, 2020.

Parotto, Giuliana. Oltre il corpo del leader. II Melangolo, 2016.

Perniola, Mario. Da Berlusconi a Monti. Mimesis, 2012.

Policastro, Gilda. "Il faut être absolument hypermoderne." Alfabeta2, 14 sett. 2014. https://www.alfabeta2.it/2014/09/14/faut-etre-absolument-hypermoderne/.

Consultato il 15 giu. 2021.

Recalcati, Massimo. L'uomo senza inconscio. Figure della nuova clinica psicoanalitica. Raffaello Cortina, 2010.

Rhéaume, Jacques. "L'hyperactivité au travail : entre narcissisme et identité." L'individu hypermoderne, sous la direction de Nicole Aubert, Érès, 2010, pp. 89-102.

Sartre, Jean-Paul. L'immaginario. Psicologia fenomenologica dell'immaginazione. 1940. Nuova edizione a cura di Raoul Kirchmayr, Einaudi, 2007.

Walton, Kendall Lewis. Mimesi come far finta. 1990. A cura di Marco Nani, Mimesis, 2011.

Weber, Max. L'etica protestante e lo spirito del capitalismo. 1905. Traduzione di Anna Maria Marietti, Rizzoli, 2013.

Gerardo landoli si è laureato in Italianistica all'Università di Bologna. Attualmente, è dottorando del CAER (Centre Aixois d'Études Romanes) presso l'Università di AixMarseille. Si occupa di rappresentazioni della violenza e del potere nella letteratura e nella serialità televisiva italiana contemporanea.

https://orcid.org/0000-0003-0662-5135

gerardo.iandoli90@gmail.com 